\title{
Design and development of sensorless based 5-DOF bilaterally controlled surgical manipulator: A prototype
}

\author{
Sakol Nakdhamabhorn, M. Branesh Pillai, Jackrit Suthakorn \\ Department of Biomedical Engineering, Faculty of Engineering, Mahidol University, Thailand
}

\begin{tabular}{l} 
Article Info \\
\hline Article history: \\
Received Jun 12, 2020 \\
Revised Nov 13, 2020 \\
Accepted Dec 5, 2020 \\
\hline
\end{tabular}

\section{Keywords:}

Bilateral control

Disturbance observer

Medical robotics

Robotic surgery

Sensorless sensing

\begin{abstract}
Minimally invasive surgery (MIS) is one of the most challenging tasks in surgical procedures due to the lack of visibility of the surgical area, instrument orientation, and depth perception. A tele-operated robot assisted minimally invasive surgery is developed to enhance a surgeon's hand dexterity and accuracy. To perform MIS, the surgeon controls a slave manipulator via a master manipulator, so the force feedback and motion feedback are required to imitate an amount of action and reaction force between master and slave manipulator. The complicated MIS requires more complex surgical manipulator with multi DOFs and multiple force feedback. The limitation of multiple DOFs force feedback is a bandwidth of torque sensors. Therefore, this study proposes a sensorless based 5-DOF bilaterally controlled surgical manipulation. In this research disturbance observer (DOB) is used to identify the internal disturbance of the system, which is used to estimate the reaction torque. This research mainly focuses on a 5-DOF bilaterally controlled surgical manipulator to maintain a position and additional force. The result of torque error in contact motion is less than $2 \%$, the non-contact motion error is not over 5\%, and it is evident that the error is always less than $0.3 \%$ for the position response.
\end{abstract}

This is an open access article under the CC BY-SA license.

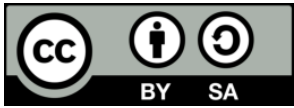

\section{Corresponding Author:}

Jackrit Suthakorn

Department of Biomedical Engineering

Faculty of Engineering, Mahidol University, Thailand

Email: jackrit.sut@mahidol.ac.th

\section{INTRODUCTION}

Surgeries are always frightening to everyone, whether it is a child or an adult. The surgeon has to make a large cut in the body for transparency so that he can perform the operation successfully, which may leave scars in the body as a 'symbol of memory'. Fortunately, by the invention of minimally invasive surgery (MIS), surgeries became more safer with limited size and number of cuts or incisions [1]. As MIS is very popular among doctors and patients, researchers have proposed many modifications to improve the technical skills of surgeons [2-5]. However, the mechanical and technical nature of the equipment limits the visibility of the organs, degree of motion, depth of the incision and hand-eye coordination in MIS. Also, loss of haptic feedback restricts the surgeon to realize a touching sensation of organs which may lead to internal injury [6,7]. Further, the primary surgical tool insertion in MIS requires high precision and micro-level accuracy on the positioning. This role of fine manipulation is limited by tremor and drift in physiology.To overcome all these human functionality limitations, robotic assistance is the best option as it is unaffected by those factors [1, 8-13].

Recently, the surgical robots that assist surgeons have become the significant focus of robotics research. Particularly, there are many interests of robotic research in common with a neurosurgery field [8-13]. In a robotic assisted MIS, there will be the master manipulator used by the surgeon to provide motion commands to the slave who will perform the actual task. The existing manipulators limit the degree of 
motion to 3-DOF which restricts the flexibility of the movement commands given in the master end. Thus, considering limitations of degree of motion in 3-DOF robotic assistance, this study proposes a sensorless based 5-DOF bilaterally controlled surgical arm.

The trouble with a bilateral controlled robotic system is that it results into an unbridled internal configuration [1, 14]. It is because it operates via both systems simultaneously and therefore, dynamic systems to be incoperate with the bilateral control system. In dynamic systems, task and posture are uncoupled and acontrolled independently, thus enhancing the operational capabilities. Bilateral control system involves the principle of action and reaction as shown in Figure 1. The slave device operated via the master device. The feedback required to the Master device requires amount of the force along with motion feedback [15-17]. The force sensor having limited bandwidth when added to the slave device transmits reaction forces [18]. For the torque sensation, the disturbance observer (DOB) is included and it is effective against disturbance compensation and is required for reaction torque estimation. The force controller determines the torque of the reaction when sensing the system's internal disturbance by the the cutoff frequency [19].

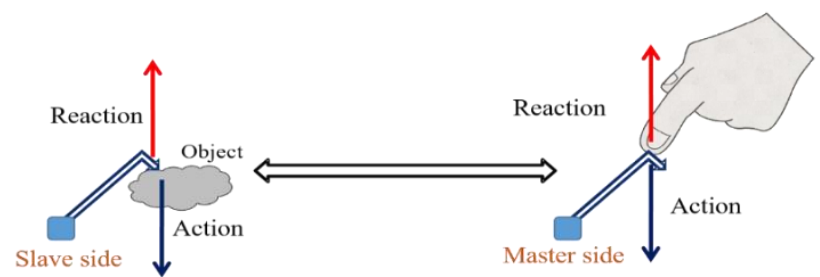

Figure 1. Action and reaction with remote sensing

Improving the operating capability of the bilaterally controlled surgical arm is the emphasis in this research. Obaid et al. [20] in the said case, the movement of the system is isolated into a compelled course and unconstrained heading. This is achieved by controlling surgical arm speed. The position, introduction and locale of the apparatus tips with respect to one another, change the speed and inner organs in the patient's midriff dependent on the robot's workspace. The specialist needs to concentrate on the objective (working point), condition (touchy tissues in the working areas) and about the crash of instruments while leading surgery. In this study, the central idea is to control the development of the surgical arm amid a neurosurgical procedure. Whereas, to maintain a strategic distance from an additional weight on the surgeon is to think whether the mechanical arm and apparatus will go past the working space or the instruments will go underneath the working parts or is there any clash between the devices and nerves or organs.

\section{RESEARCH METHOD}

\subsection{DC motor model}

Applying Kirchoff's Voltage Law to the DC motor equivalent circuit, shown in Figure 2.

$$
V_{a}=L \frac{d I_{a}^{r e f}}{d t}+R I_{a}^{r e f}+e_{b}
$$

and,

$$
\begin{aligned}
& e_{b}=k_{e} \omega \\
& T_{m}=k_{t} I_{a}^{r e f}
\end{aligned}
$$

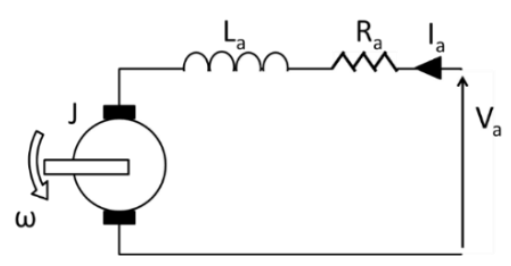

Figure 2. DC motor equivalent circuit diagram 
The control block diagram of the PMDC motor depicted in Figures 3 and 4 where the motor torque expressed as,

$$
T_{m}=J \frac{d \omega}{d t}+T_{f}+B \omega+T_{l}
$$

where,

$$
\begin{aligned}
& J \quad=\text { Inertia of the motor } \\
& B=\text { Viscous friction coefficient } \\
& T_{l}=\text { Load torque } \\
& T_{f}=\text { Static friction torque } \\
& \omega=\text { Angular speed } \\
& k_{t}=\text { Torque constant }
\end{aligned}
$$

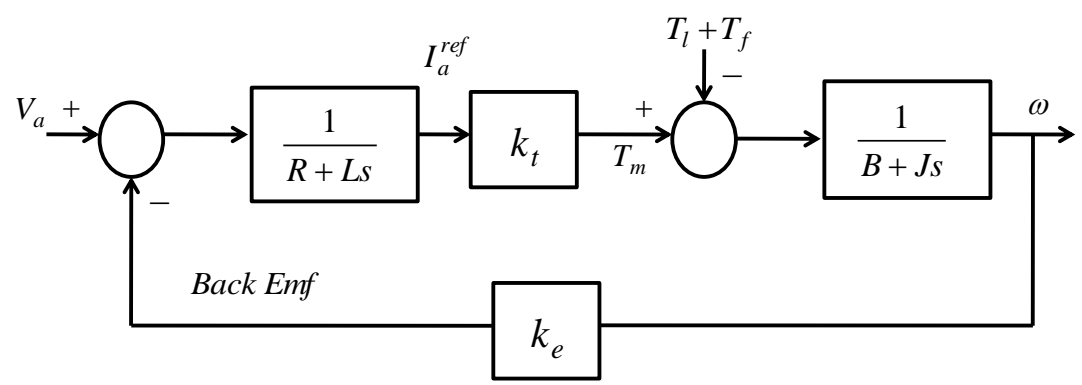

Figure 3. Block diagram of DC motor

The (4) expressed as,

$$
\begin{aligned}
& J \ddot{\theta}=T_{m}-T_{L} \\
& J \ddot{\theta}=K_{t} I_{a}^{r e f}-T_{L}
\end{aligned}
$$

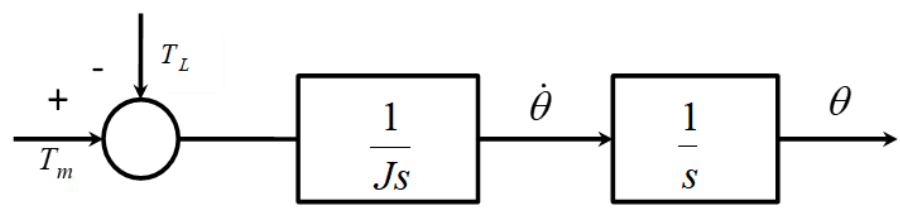

Figure 4. Block diagram of a servo motor

where, $T_{L}$ mentioned in the Figure 4 is a sum of external torque, inertia torque, and torque generated from viscous and coulomb friction.

$$
T_{L}=T_{\text {int }}+T_{\text {ext }}+\left(T_{f}+B \dot{\theta}\right)
$$

where,

$$
\begin{aligned}
& T_{i n t}=\text { Inertia torque and gravity effect } \\
& T_{\text {ext }}=\text { External torque } \\
& T_{f}=\text { Static friction } \\
& B \dot{\theta}=\text { Viscous friction }
\end{aligned}
$$

The reference current $I_{a}^{r e f}$ is the input for the motor and PWM voltage pulses are responsible for the control of the motor. For correct functionality, $T_{L}$ must be compensated, as the motor torque $T_{L}$ 
cannot be directly controlled using $I_{a}^{r e f}$. As the torque $T_{L}$ disturbs the output torque, it is also termed as 'Disturbance Torque'. Hence, 'Disturbance Observer' is introduced to measure this disturbance torque and compensate it [19].

\subsection{Disturbance observer modelling}

The (4) consists of a couple of parameters torque constant and motor inertia, which can be changed because of several properties. Similarly, because of the mechanical configuration of the motion system, the inertia might also change. In the case of the torque coefficient, it changes with respect to the rotor position of the electric motor, which causes the irregularity in the distribution of the magnetic flux throughout the surface of the rotor $[15,19]$.

$$
\begin{aligned}
& J=J_{n}+\Delta J \\
& K_{t}=K_{t n}+\Delta K_{t}
\end{aligned}
$$

where;

$$
\begin{array}{ll}
J_{n} & =\text { Nominal inertia } \\
\Delta J & =\text { Inertia variation } \\
K_{t n} & =\text { Nominal torque coefficient } \\
\Delta K_{t} & =\text { Variation of torque coefficient }
\end{array}
$$

By substituting (8) and (9) into (4), (10) is obtained.

$$
\left(J_{n}+\Delta J\right) \ddot{\theta}=\left(K_{t n}+\Delta K_{t}\right) I_{a}^{r e f}-T_{l}
$$

By rearranging,

$$
J_{n} \ddot{\theta}=K_{t n} I_{a}^{r e f}-\left(T_{l}+\Delta J \ddot{\theta}-\Delta K_{t} I_{a}^{r e f}\right)
$$

where;

$$
T_{d i s}: \text { Disturbance torque }
$$

Therefore,

$$
T_{\text {dis }}=T_{\text {int }}+T_{\text {ext }}+(f+D \dot{\theta})+\Delta J \ddot{\theta}-\Delta K_{t} I_{a}^{\text {ref }}
$$

Apparently, the disturbance torque comprises all the external torques, internal torques, friction torques and torques due to parameter variations and all of them compensated so that the accurate output torque obtained. From (11) $T_{\text {dis }}$ can be calculated as (13):

$$
T_{\text {dis }}=K_{t n} I_{a}^{r e f}-J_{n} \ddot{\theta}
$$

The unknown value of $T_{d i s}$ can be calculated using the known values at the right-hand side in the (13). Using the velocity response $\dot{\theta}$ and the torque current $I_{a}^{\text {ref }}$, the estimated disturbance torque $\widehat{T}_{d i s}$ is obtained. As shown in (14), the estimation is done through a first-order low-pass filter, where $g_{\text {dis }}$ represents the cut-off frequency of the low-pass filter. Low-pass filter to filter the noises generating from the differentiation [20].

$$
\widehat{T}_{d i s}=\frac{g_{\text {dis }}}{s+g_{\text {dis }}} T_{\text {dis }}
$$

The (14) represented in the form of a block diagram. In (12) and (13) demonstrated as a block diagram as shown in Figure 5. This structure comprises of a differentiator, which will escalate the noise. The implementation of the disturbance observer in the DC motor observed in the following Figure 6. 


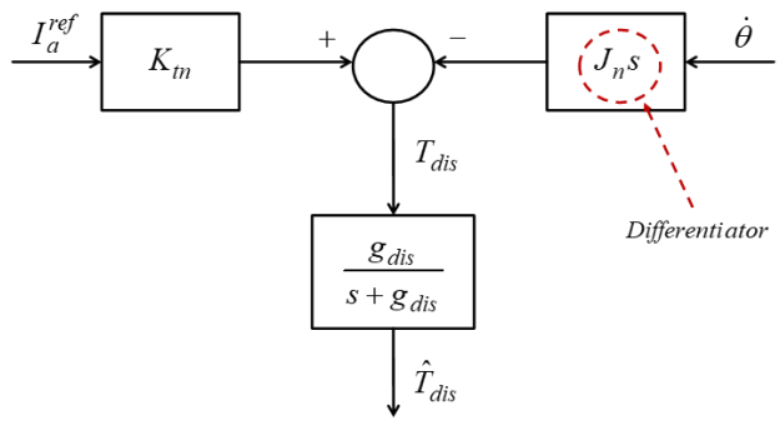

Figure 5. Estimated disturbance torque through a low pass filter

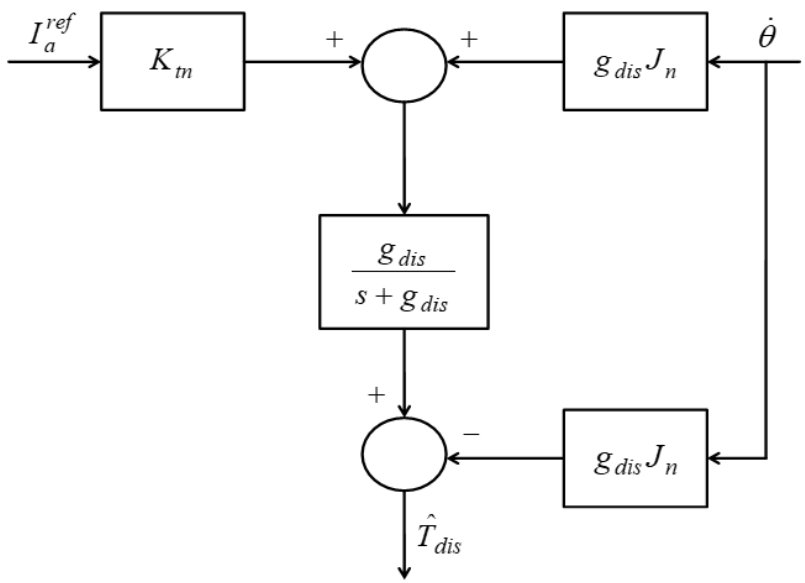

Figure 6. Block diagram of the disturbance observer

\subsection{Reaction torque observer modeling}

The significance of the disturbance detected in practical application is observed as disturbance compensation as well as in the reaction torque estimation [1,21]. Without using a torque sensor, the disturbance observer can identify the internal disturbance of the system and also estimate the reaction torque as shown in Figure 7. It is well accounted that their relationship of action and reaction is determined by the transmission of force sensation within bilateral control. In this case the disturbance observer starts prompt calculation and estimation of the reaction torque by increasing the cut-off frequency. The reaction torque observer is considered as a modification of the disturbance observer.

$$
T_{\text {dis }}=T_{\text {int }}+T_{\text {ext }}+(f+D \dot{\theta})+\Delta J \ddot{\theta}-\Delta K_{t} I_{a}^{\text {ref }}
$$

The external torque acting on the slave manipulator is considered as the Reaction torque and it calculated as:

$$
T_{\text {ext }}=T_{\text {dis }}-\left[T_{\text {int }}+(f+D \dot{\theta})+\Delta J \ddot{\theta}-\Delta K_{t} I_{a}^{\text {ref }}\right]
$$

Since at the moment of contact,

where;

$$
T_{\text {ext }}=T_{\text {react }}
$$

$T_{\text {react }}:$ Reaction Torque

$$
T_{\text {react }}=T_{\text {dis }}-\left[T_{\text {int }}+(f+D \dot{\theta})+\Delta J \ddot{\theta}-\Delta K_{t} I_{a}^{r e f}\right]
$$

The block diagram of the reaction torque observer is shown in Figure 8. 


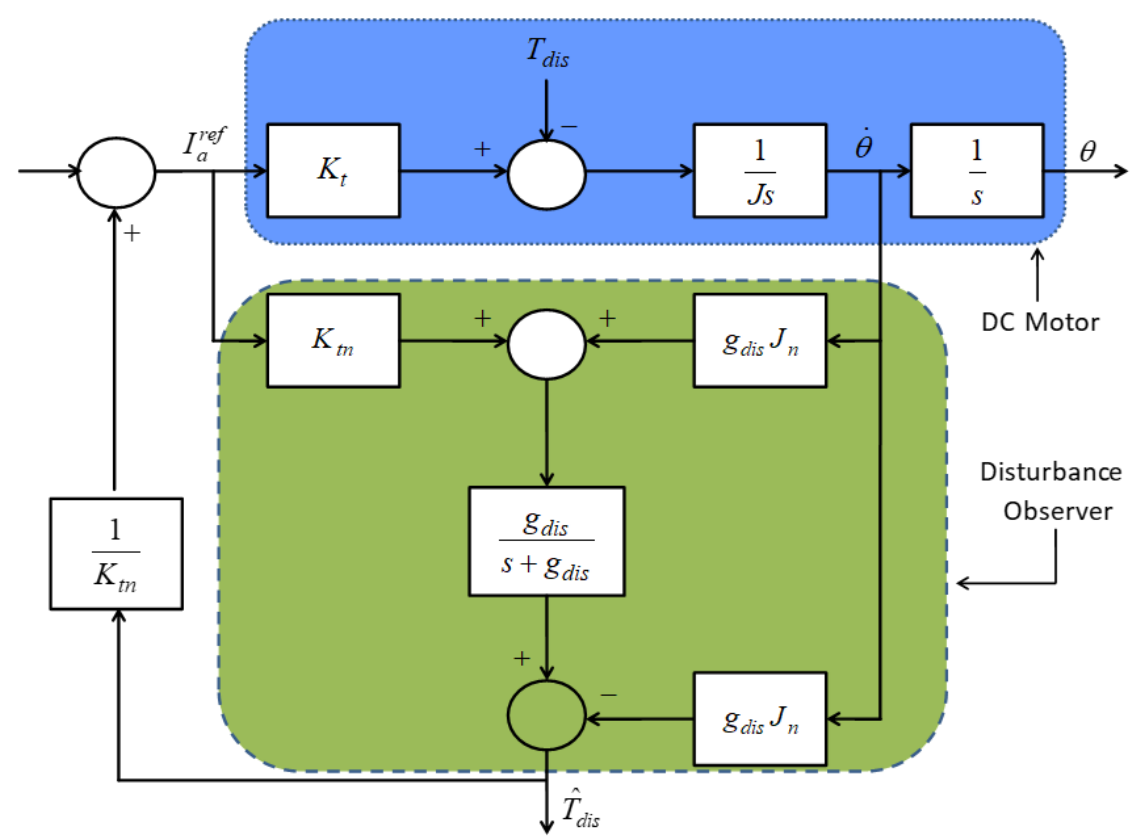

Figure 7. Disturbance observer with the DC motor

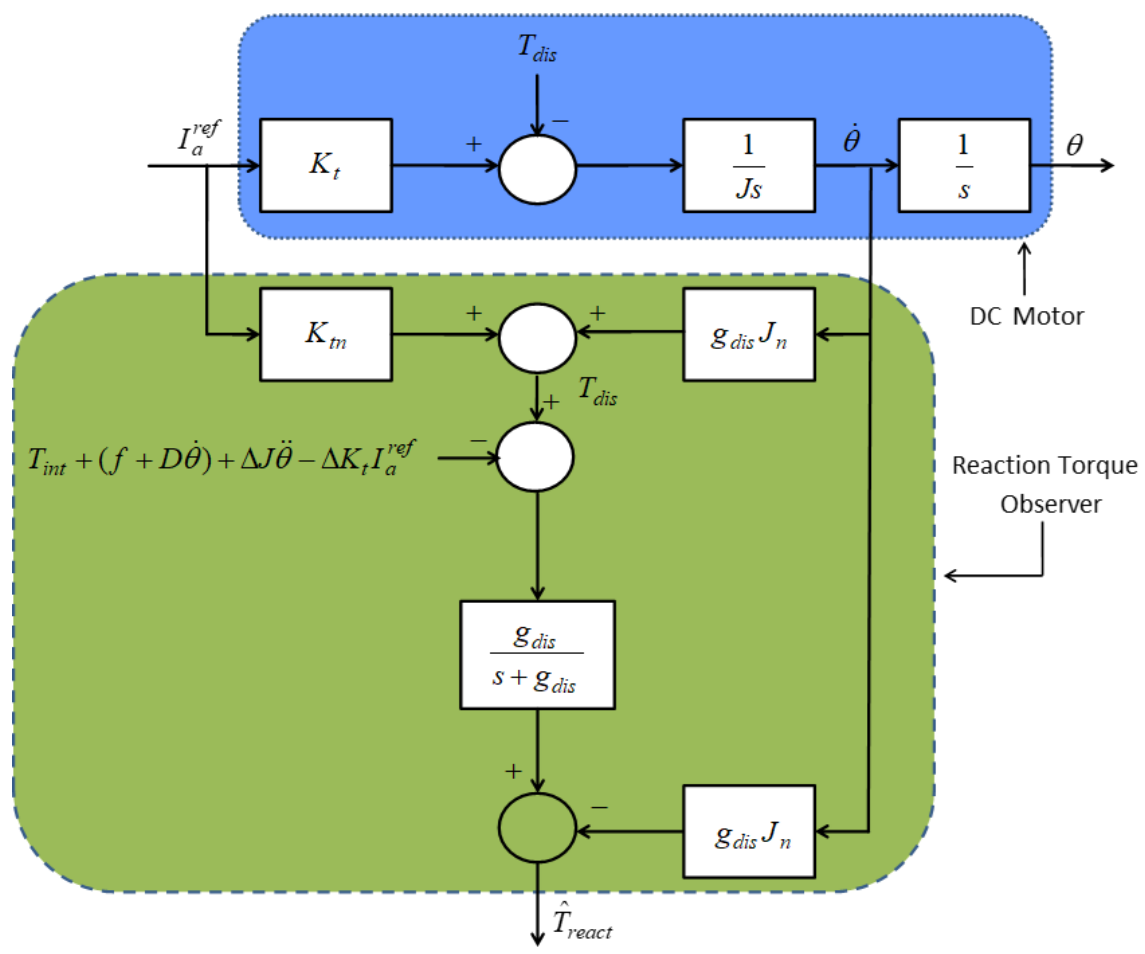

Figure 8. Reaction torque observer with the DC motor

The disturbance observer offers numerous advantages including observation of disturbance torque and compensation. While a regular force sensor consists of narrow bandwidth, the disturbance observer's offers relatively wider bandwidth [22]. The force sensor consisting of narrow bandwidth cannot offer substantial force sensation and is just able to detect wherever it is implemented. Due to being to operate under a smaller sampling time, which increases the observer gain as much as possible, the disturbance observer can provide a better alternative with the wider bandwidth. As such, the disturbance observer is relatively more appropriate for transmission of force sensation in comparison to the force sensors. 


\subsection{Bilateral control modeling}

The bilateral controller controls the responses from both, the master as well as the slave and converts it into inputs for each other. To control the slave side, torque/position information from the master side is used and vice versa. Thus, it is important to capture the exact torque/position information of the slave to provide high transparency to the master side $[17,22]$. However, according to control theory, transparency and stability cannot achieve simultaneously for bilateral controllers due to the system and environment uncertainties. Therefore, a balance is fetched by the bilateral system between transparency and stability. In a bilateral controller, force sensors used to detect the force of master as well as the slave and the external forces. Strain gauges are one of the common force sensors used in the bilateral controller. However, these force sensors have narrow bandwidth and when it is in contact with the motion, the accuracy of the output depreciates. In addition, the force sensors can detect only the force around it. Thus, they cannot provide a vivid sensation of the force, as it is very difficult to fix the sensors in the places where the force to be measured. To overcome these drawbacks, disturbance observer can be used as a force sensor that can provide much wider bandwidth and possible to set the sampling time short and the observer gain high. Ohnishi et al. [16] have successfully analyzed the bandwidth of force control based on RTOB and the frequency bandwidth of sensed force $(0-80 \mathrm{~Hz})$. Though due to the resource constraints of the microcontroller to provide higher bandwidth and the human interaction with the system is producing lower frequencies, the researcher has used lower bandwidth $(0-40 \mathrm{~Hz})$ for the study purpose.

The input to the bilateral system includes not only the force but also the elements of disturbance from the environment [1,23]. To avoid this unwanted addition to the input, a good disturbance rejection mechanism is bringing together. Further, through the disturbance observer, robust motion control with bilateral control is recognized. For the realization of these objectives, acceleration control is essential. The structure of bilateral control is resultant from the law of action and reaction.

Therefore, when the operator manipulates the master, the slave has to be in the same environment. Otherwise, the scaling has to be auxiliary to the input. The following (19) and (20) represent bilateral control without scaling in which subscript $m$ denotes master and $s$ denotes slave.

$$
\begin{aligned}
& \theta m-\theta s=0 \\
& \tau m+\tau s=0
\end{aligned}
$$

Both (19) and (20) are transformed into acceleration to attain ideal force and position control, which is necessary for a bilateral control.

$$
\begin{aligned}
& \theta m-\theta s=0 \\
& \theta m+\theta s=0
\end{aligned}
$$

As the disturbance observer is also used as a force sensor, the sampling time should be as small as possible to get a better response [24, 25]. The (21) is known as differential mode, which represents the position relationship and (22) is known as common mode between the master and slave system, which represents the acceleration and attributed to the sign of equations. Hence, if the force and position combined in the acceleration dimension, it is possible to achieve independent control of both of them in a bilateral system. Thus, the (21) and (22) are transformed into the following to get the optimal goal of bilateral control as shown in Figure 9.

$$
\begin{aligned}
& \theta m-\theta s=\theta \text { com } \rightarrow 0 \\
& \theta m+\theta s=\theta \text { dif } \rightarrow 0
\end{aligned}
$$

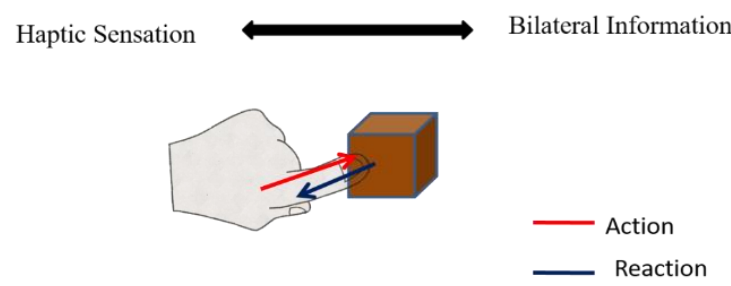

Figure 9. Bilateral control representation 


\subsection{5-DOF bilaterally controlled surgical arm modelling}

A bilateral 5- DOF robotic system is developed in this research to perform surgical procedures by taking into consideration the workspace and the operational movements. The system consists two main units: the master manipulator and the slave manipulator with same dimension. The manipulator has 5 degree-offreedom (DOF) with five actuators and three links. The end-effector of the slave manipulator attached with the surgical tool, which can carry $0.5 \mathrm{~kg}$. The manipulator is implemented using ROBOTIS smart actuator model XM430-W350 and XM540-W270. The actuator is controlled by PID position and velocity control and provides feedback on the real-time position, velocity as well as torque. Robot operation system (ROS) platform is used as the software control system and user interface. Figure 10 shows the conceptual design of manipulator and Figure 11 is the completed manipulator.

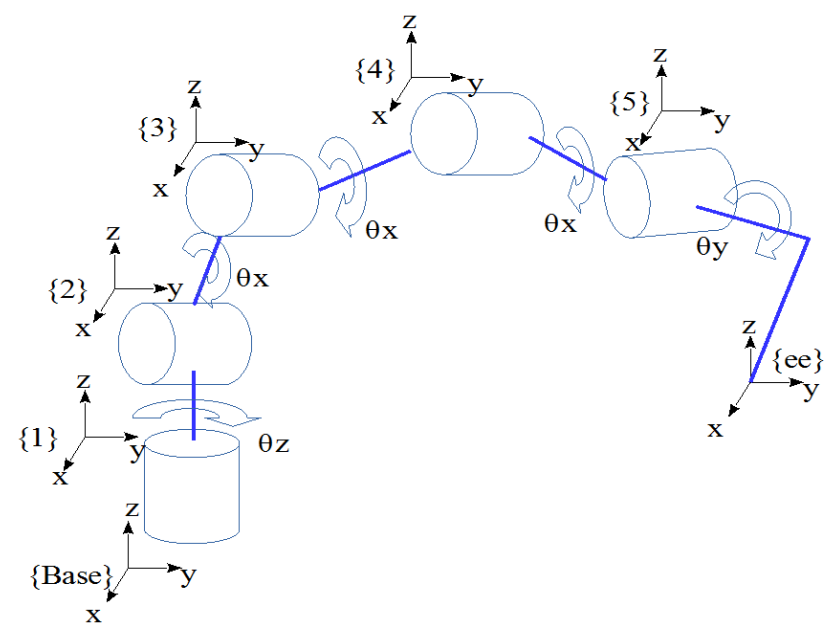

Figure 10. Conceptual design of 5-DOF manipulator
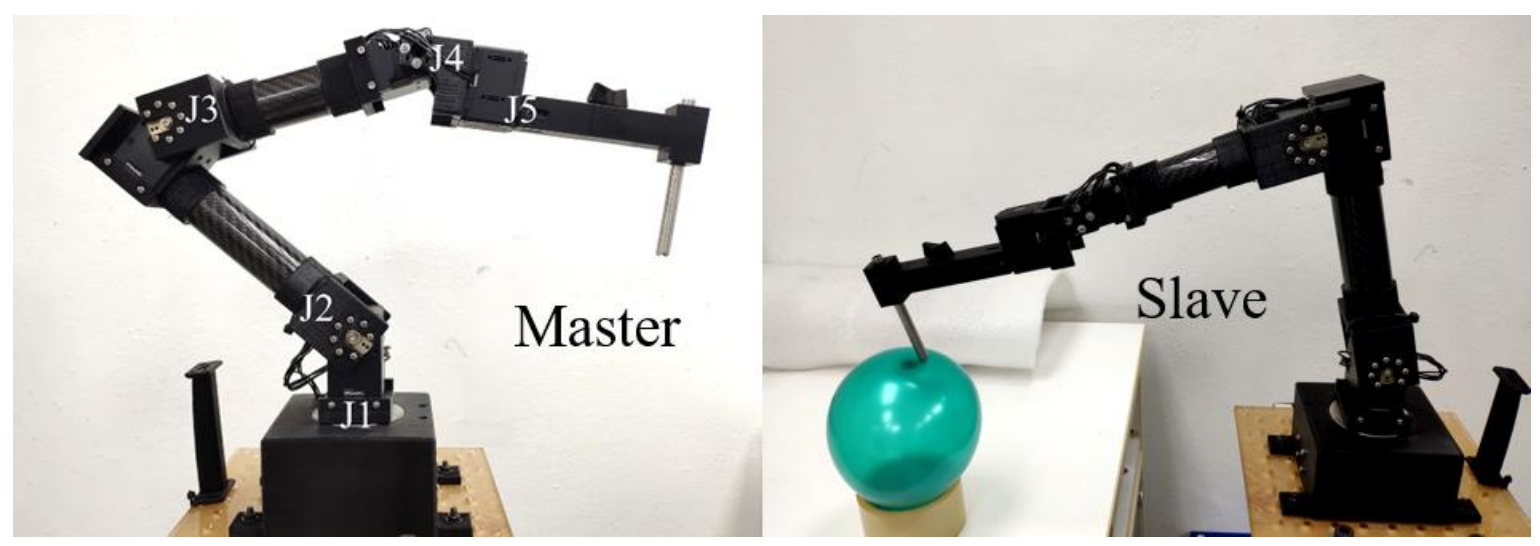

Figure 11. Bilaterraly controlled 5-DOF manipulator

To command the manipulator, a kinematic relationship is used to generate forward kinematic and inverse kinematic of the robotic manipulator. The forward kinematic transforms the joints coordinate $(\varnothing)$ to the Cartesian coordinate $(\mathrm{x}, \mathrm{y}, \mathrm{z})$. Therefore, the manipulator moves on the Cartesian coordinate $(\mathrm{x}, \mathrm{y}, \mathrm{z})$ by commanding a joint angle in each joint actuator. The inverse kinematic transforms a Cartesian coordinate (x, $\mathrm{y}, \mathrm{z})$ to a joint coordinate $(\varnothing)$. Therefore, the operator commands position and orientation on the Cartesian coordinate to control the manipulator joint's movement.

Figure 10 represents the homogeneous relationship of the manipulator joint coordinate, which is assembled from seven joint coordinates. The base of the manipulator is a fix reference coordinate ( $\{$ Base $\})$ of the system. The $\{1\}$ coordinate is a revolute joint rotated in $\mathrm{z}$-axis, $\{2\},\{3\},\{4\}$ in $\mathrm{X}$-axis and $\{5\}$ in $\mathrm{Y}$-axis. The tip of the surgical tool is a fixed end of the effector coordinate (\{ee\}). The kinematic of the manipulator is calculated based on the homogeneous transformation of the joint coordinate's relationship shown in (25). 


$$
\underset{\text { Base }}{e e} H=\underset{\text { Base }}{1} H *{ }_{1}^{2} H *{ }_{2}^{3} H *{ }_{3}^{4} H *{ }_{4}^{5} H *{ }_{5}^{e e} H
$$

\section{RESULTS AND DISCUSSION}

\subsection{Experiment setup}

The experiment setup was set as short distance teleoperation with a bilaterally controlled masterslave system. The master manipulator is installed at the user station and the slave manipulator is placed on an experimental environment. Master and slave manipulators are the main units of this experiment in which the master side involves human interaction. For an ideal brake by wire control, the position and the torque applied at the master end should appear the same as in the slave end. In this experiment, the slave is not in contact with any material so that it can move freely. To conduct a contact motion test, the slave is brought into an environment where the manipulator comes in contact with an object (Rubber). In the functional world the human tissue's tensile strength varies from patient to patient, hence the experiments were conducted with materials of rubber types (with an unknown tensile strength value). The experiments were performed under the supervision of a Surgeon from Siriraj Hospital, Mahidol University as shown in Figure 12. In order to check the stability of the proposed system, the selected material was utilized and it was found that this system can be used universally for robot-assisted neurosurgery. The experiments were performed with an observer gain (force bandwidth of $0-80 \mathrm{rad} / \mathrm{sec}$ ). In these experiments, the two different values of gains were chosen $40 \mathrm{~Hz}$ in Figure 13 and $80 \mathrm{~Hz}$ in Figure 14. The response of torque and position is shown in Figures 13 and 14 for both contact and non-contact motion.

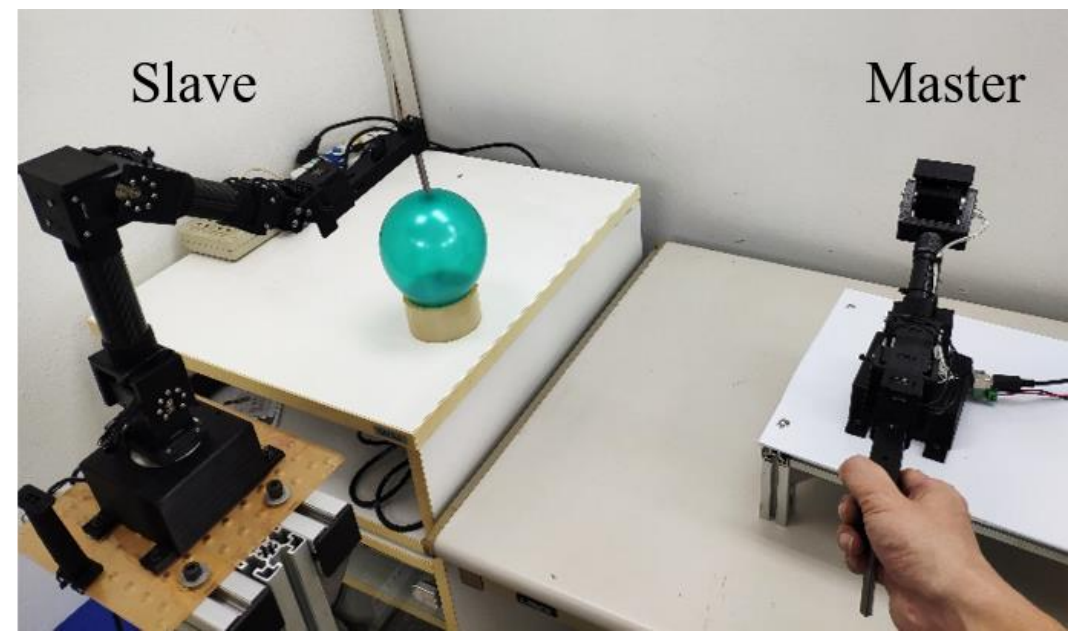

Figure 12. Experimental setup of bilaterally controlled master-slave system

From the Figures 13 and 14, it is evident that the slave manipulator is closely following the position response of the master manipulator with insignificantly smaller position errors as shown in Figure 13 (a), (c), (e), (g) and Figure 14 (a), (c), (e), (g) (maximum position error is less than $0.3 \%$ ). Figure 13 (i), (j) and Figure 14 (i), (j) shows the position response and torque response of end effector (joint 5) is slightly different from the other joints because of grasping motion. Figure 13 (b), (d), (f), (h) and Figure 14 (b), (d), (f), (h) shows the torque responses of the master and the slave manipulators in different cut-off frequencies $40 \mathrm{~Hz}$ and $80 \mathrm{~Hz}$, which is exactly the mirror images of each other. The slave manipulator closely follows the torque applied in the master manipulator, see Figures 13 and 14. When Figures 13 and 14 is compared, high frequency variations of the torque can be observed at a higher cut-off frequency.

In contact motion, the response of slave manipulator follows as that of the torque applied in the master end, see Figures 13 and 14. An error bar is also generated on the top of the torque curve of the "master" as shown in Figure 15 (a). So, the test result shows that the torque error in contact motion is less than $2 \%$ whereas it is less than $5 \%$ in non-contact motion.

Figures 14 (a), (c), (e), (g) and Figure 13 (a), (c), (e), (g) indicates that the position response of the slave is similar to that of the master in contact motion. Even though the test shows considerable error in position response due to the low proportional gain selected in the controller, it should not be increased because of the torque limits of the motor. From the error bar, see Figure 15 (b), it is evident that the error is always less than $0.3 \%$ for the position response. 

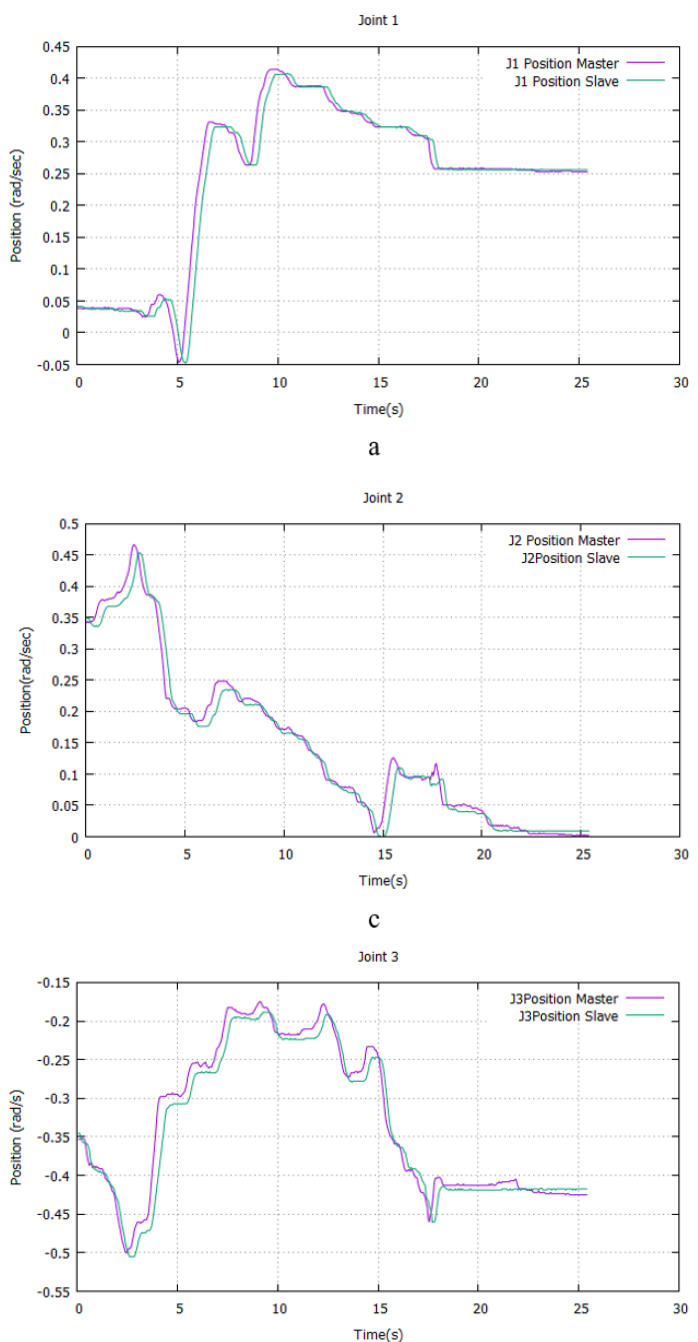

e
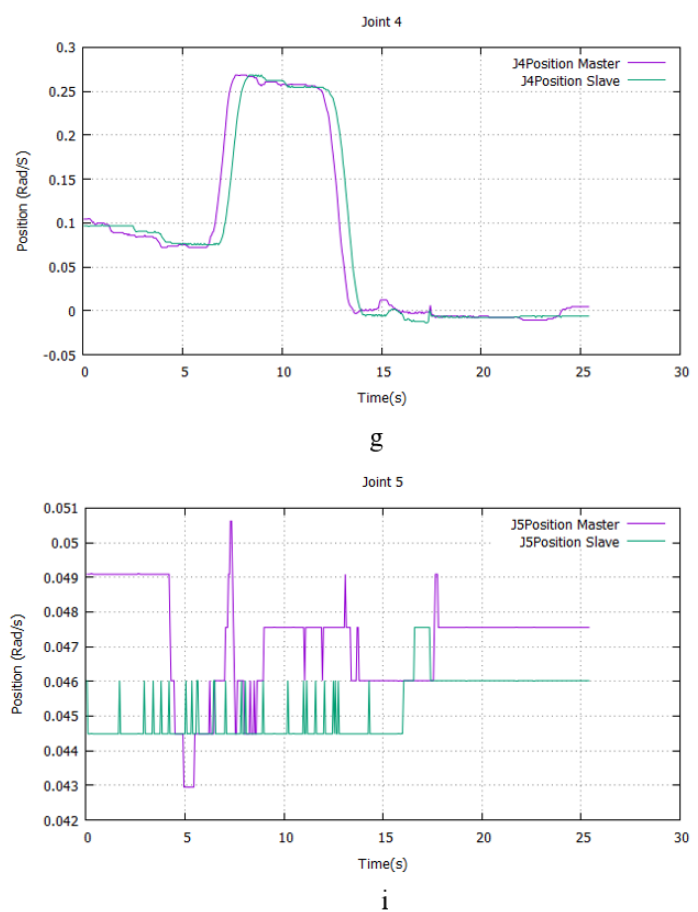

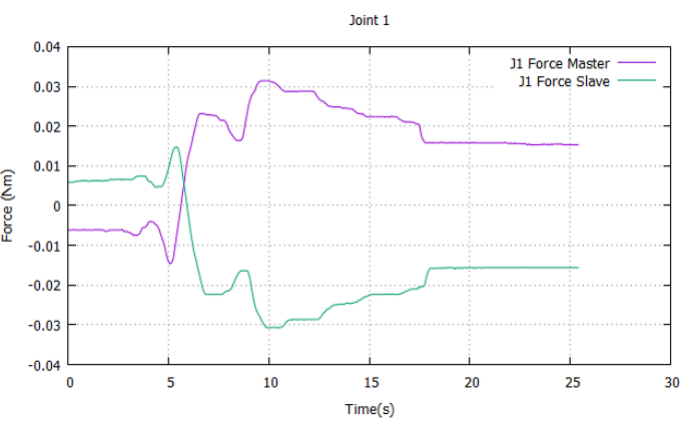

b

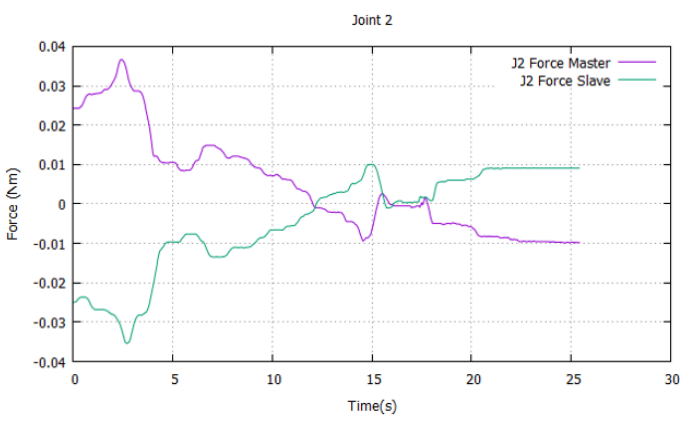

d

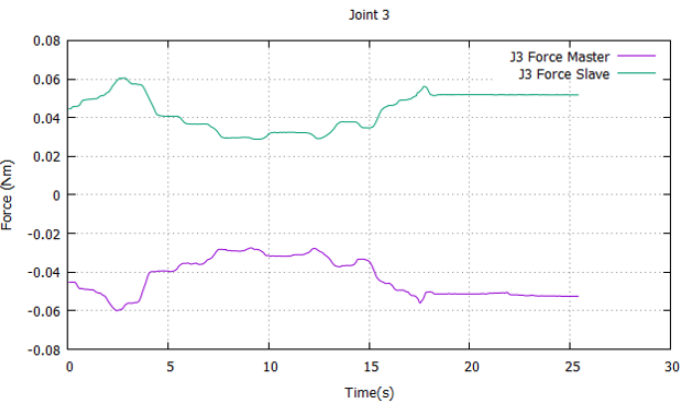

f

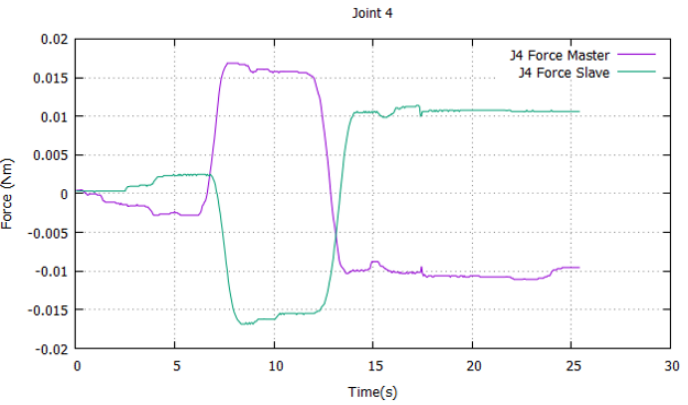

h

Joint 5

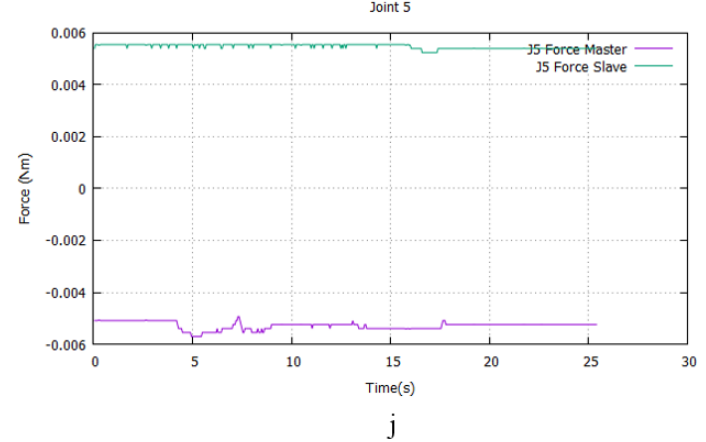

Figure 13. Position and torque response for all the joint $\left(\mathrm{J}_{1}-\mathrm{J}_{5}\right)$ with observer gain $\mathrm{G}_{\mathrm{dis}}=40 \mathrm{~Hz}$ 

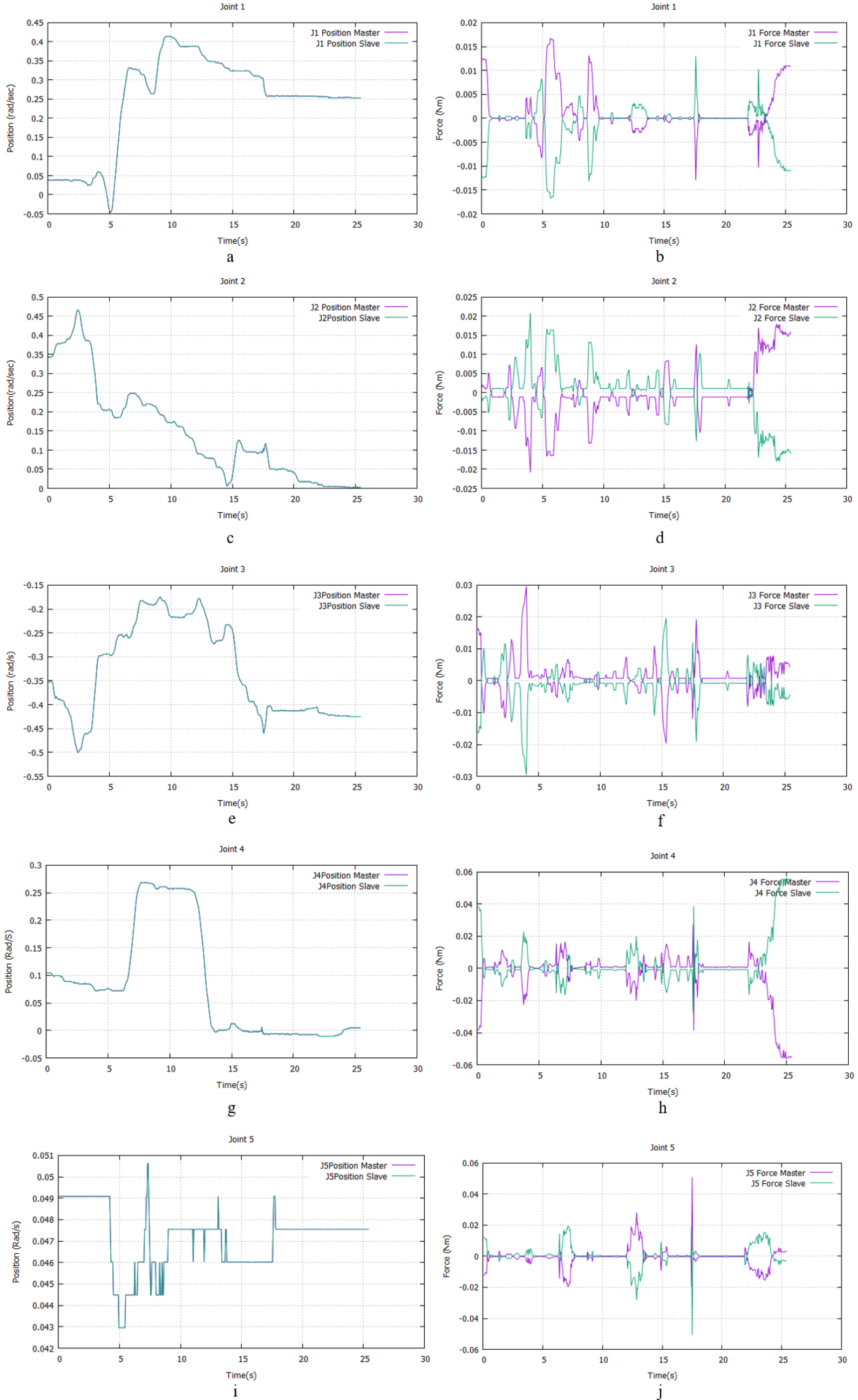

Figure 14. Position and torque response for all the joint $\left(\mathrm{J}_{1}-\mathrm{J}_{5}\right)$ with observer gain $\mathrm{G}_{\mathrm{dis}}=80 \mathrm{~Hz}$ 

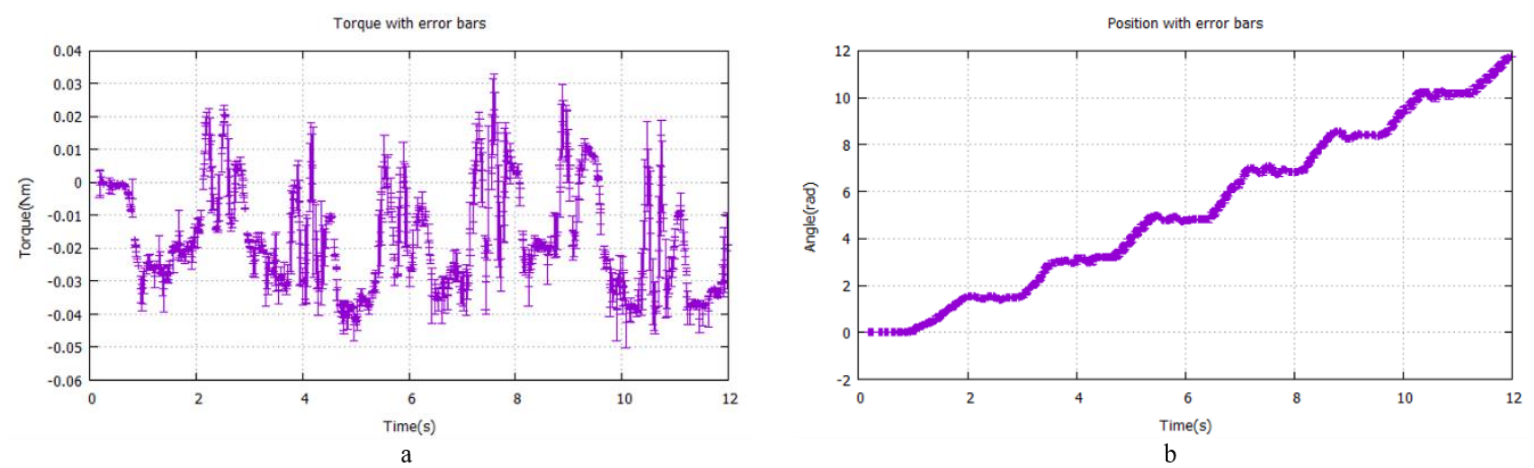

Figure 15. Averaged error response for joints $\left(\mathrm{J}_{1}-\mathrm{J}_{5}\right) \mathrm{G}_{\mathrm{dis}}=80 \mathrm{~Hz}$, (a) Averaged position error, (b) Averaged torque error

\section{CONCLUSION}

The applications of Minimal invasive surgery are increasing day by day, and now surgery is not limited to only neurosurgery. In the present situation the surgeons are not excluded from the surgical environment, without even considering the safety factors. Therefore, feedback in MIS is a technology that has not yet arrived on commercial MIS platforms. But due to current interests and in-depth research it will eventually lead to the development of better MIS robots in the future. Even though the bilateral control concept was initialized long ago and it is currently being implemented by various researchers all around the world, its operationality improvement is not yet developed significantly; hence the initiative was arisen to implement operationality improvement. In this study a sensorless based 5-DOF bilaterally controlled surgical manipulation was proposed. The DOB is implemented to identify the internal disturbance of the system, which is used to estimate the reaction torque. The goal is to bileratlly controlled a 5-DOF surgical manipulator to maintain a postion and additional force while a surgeon preforms in MIS operation. The position and torque responses were consummate and obtained distinctly for both contact and non-contact conditions. During the non-contact condition the position responses of slave manipulator follows the master system very closely. A lower torque error shows during the contact motion and the error could be reduced by introducing nonlinear control strategies in particular adaptive fuzzy hierarchical sliding mode control for a category of MIMO nonlinear time-delay systems with enter saturation.

\section{ACKNOWLEDGEMENTS}

The National Research University Funds through Mahidol University and Government Research Budget through Mahidol University (Grant No. 111-2558) financially support this research. Another fund resource by the Computer-Integrated Intelligent Medical System Project under the Thailand National Research University Grant through Mahidol University, Thailand. Moreover, we would like to thank Clinical Professor Areesak Chotivichit from Siriraj Hospital for their valuable knowledge and recommendations towards the development of this system and BART LAB Researchers for their kind support.

\section{REFERENCES}

[1] B. M. Pillai, C. Wilasrusmee, and J. Suthakorn, "Observer based dynamic control model for bilaterally controlled MU-LapaRobot: surgical tool force limiting," International Journal of Electrical and Computer Engineering, vol. 10, no. 1, pp. 828-839, Feb. 2020.

[2] A. Onibokun and L. T. Holly, "Minimally invasive pedicle screw fixation," Operative Techniques in Neurosurgery, vol. 7, no. 2, pp. 72-78, 2004.

[3] F. Ringel, M. Stoffel, C. Stüer, and B. Meyer, "Minimally invasive transmuscular pedicle screw fixation of the thoracic and lumbar spine," Neurosurgery, vol. 59, no. 4 Suppl 2, pp. 361--67, 2006.

[4] R. J. Mobbs, P. Sivabalan, and J. Li, "Technique, challenges and indications for percutaneous pedicle screw fixation," Journal of Clinical Neuroscience, vol. 18, no. 6, pp. 741-749, 2011.

[5] K. T. Foley and S. K. Gupta, "Percutaneous pedicle screw fixation of the lumbar spine: preliminary clinical results," Journal of Neurosurgery: Spine, vol. 97, no. 1, pp. 7-12, 2002.

[6] I. D. Gelalis, N. K. Paschos, E. E. Pakos, A. N. Politis, C. M. Arnaoutoglou, A. C. Karageorgos, A. Ploumis, and T. A. Xenakis, "Accuracy of pedicle screw placement: A systematic review of prospective in vivo studies comparing free hand, fluoroscopy guidance and navigation techniques," European Spine Journal, vol. 21, no. 2, pp. 247-255, 2012.

Bulletin of Electr Eng \& Inf, Vol. 10, No. 2, April 2021 : 619-631 
[7] P. Cappabianca, L. M. Cavallo, A. Colao, and E. de Divitiis, "Surgical complications associated with the endoscopic endonasal transsphenoidal approach for pituitary adenomas," Journal of Neurosurgery, vol. 97, no. 2, pp. 293-298, 2002.

[8] W. J. Beutler, W. C. Peppelman, and L. A. DiMarco, "The da Vinci robotic surgical assisted anterior lumbar interbody fusion: technical development and case report," Spine, vol. 38, no. 4, pp.356-363, 2013.

[9] R. Taylor, P. Jensen, L. Whitcomb, A. Barnes, R. Kumar, D. Stoianovici, P. Gupta, Z. Wang, E. deJuan, and L. Kavoussi, "A steady-hand robotic system for microsurgical augmentation," The International Journal of Robotics Research, vol. 18, no. 12, pp. 1201-1210, 1999.

[10] S. Chumnanvej, B. M. Pillai, and J. Suthakorn, "Surgical robotic technology for developing an endonasal endoscopic transsphenoidal surgery (EETS) robotic system," The Open Neurology Journal, vol. 13, no. 1, pp. 96106, 2019.

[11] S. Chumnanvej, S. Chalongwongse, B. M. Pillai, and J. Suthakorn, "Pathway and workspace study of endonasal endoscopic transsphenoidal (EET) approach in 80 cadavers," International Journal of Surgery Open, vol. 16, pp. 22-28, 2019.

[12] M. Lefranc and J. Peltier, "Evaluation of the ROSA ${ }^{\mathrm{TM}}$ spine robot for minimally invasive surgical procedures," Expert Review of Medical Devices, vol. 13, no. 10, pp. 899-906, 2016.

[13] R. H. Taylor, A. Menciassi, G. Fichtinger, and P. Dario, "Medical robotics and computer-integrated surgery," Springer Handbook of Robotics, pp. 1657-1684, 2016.

[14] A. M. H. S. Abeykoon and K. Ohnishi, "Virtual tool for bilaterally controlled forceps robot-for minimally invasive surgery," International Journal of Medical Robotics and Computer Assisted Surgery, vol. 3, no. 3, pp. 271-280, 2007.

[15] A. Sabanovic and K. Ohnishi, "Motion control systems," John Wiley \& Sons, 2011.

[16] K. Ohnishi, S. Katsura and T. Shimono, "Motion control for real-world haptics," IEEE Industrial Electronics Magazine, vol. 4, no. 2, pp. 16-19, June 2010.

[17] A.M. Harsha S. Abeykoon, K. Ohnishi. "Realization of virtual master manipulator using bilateral control," 2006 4th IEEE International Conference on Industrial Informatics, IEEE, pp.833-838, Aug. 2006.

[18] S. A. Ali, M. F. Miskon, A. Z. Hj Shukor, and M. Q. Mohammed, "The effect of parameters variation on bilateral controller," International Journal of Power Electronics and Drive System, vol. 9, no. 2, pp. 648-659, 2018.

[19] B. M. Pillai and J. Suthakorn, "Motion control applications: observer based DC motor parameters estimation for novices," International Journal of Power Electronics and Drive System, vol. 10, no. 1, pp. 195-201, 2019.

[20] M. A. M. Obaid, A. R. Husain, and A. A. M. Al-kubati, "Robust backstepping tracking control of mobile robot based on nonlinear disturbance observer," International Journal of Electrical and Computer Engineering, vol. 6, no. 2, pp. 901-908, 2016.

[21] N. S. Hasan, N. Rosmin, S. A. Khalid, Dygku. A. Awg. Osman, Baharuddin Ishak, A. H. Mustaamal, "Harmonic suppression of shunt hybrid filter using LQR-PSO based," International Journal of Electrical and Computer Engineering, vol. 7, no. 2, pp. 869-876, 2017.

[22] A. M. H. S. Abeykoon and K. Ohnishi, "Improvement of tactile sensation of a bilateral forceps robot by a switching virtual model," Journal Advanced Robotics, vol. 22, no. 8, pp. 789-806, 2008.

[23] C. Rossa, N. Usmani, R. Sloboda, and M. Tavakoli, "A hand-held assistant for semi automated percutaneous needle steering," IEEE Trans. Bio. Eng., vol. 64, no. 3, pp. 637-648, 2017.

[24] M. Tonutti, D.S. Elson, G. Yang, A.W. Darzi, and M. H. Sodergren, "The role of technology in minimally invasive surgery: state of the art, recent developments and future directions," Postgraduate Medical Journal, vol. 93, no. 1097, pp. 159-167, 2017.

[25] R. M. M. Ruwanthika and A. M. H. S. Abeykoon, "Simulation of enhanced force limiting gripper for bilateral teleoperation," 7th International Conference on Information and Automation for Sustainability, pp. 1-6, 2014. 